\title{
ARTICLE
}

\section{Bulk shielding for laser research centre ELI Beamlines}

\author{
Veronika Olšovcováa $^{\mathrm{a}}$, Richard Haley ${ }^{\mathrm{b}}$, Lewis MacFarlene ${ }^{\mathrm{b}}$, Bedřich Rus ${ }^{\mathrm{a}}$ and Mike Griffiths ${ }^{\mathrm{a}}$ \\ ${ }^{a}$ Institute of Physics, Academy of Sciences of the Czech Republic, Na Slovance 2, Prague 8, Czech Republic; \\ ${ }^{b}$ Nuclear Technologies, plc., United Kingdom
}

\begin{abstract}
The civil structure for the laser research centre ELI Beamlines has been designed to provide adequate radiation protection for both personnel and the public. The facility will host primary sources of photons, electrons and protons. Pulsed mixed fields of high energy particles (up to several $\mathrm{GeV}$ ) will be generated, producing up to $10^{12}$ particles of primary radiation, in pulses of several fs length. Shielding calculations were undertaken using Monte Carlo transport codes FLUKA and MCNPX, together with a discrete ordinates code ATTILA. Integration of the Monte Carlo and discrete ordinates methods allowed assessment of complex shielding geometries to proceed faster than could be achieved through the use of either biased or analogue Monte Carlo alone. It was possible to design the building, so that the expected annual dose accrual of workers will be lower than $1 \mathrm{mSv}$. The main contributors to the total dose are neutrons. Therefore, for penetrations, it is necessary to ensure shielding against neutrons.
\end{abstract}

Keywords: laser acceleration; shielding design; Monte Carlo simulation; discrete ordinates code; high energy radiation; mixed field

\section{Introduction}

Recent developments in laser systems have resulted in the ability to focus ultra-short high-intensity pulses onto targets. As these intense lasers can generate ionizing radiation, the civil structure of the facilities needs to be designed to provide sufficient shielding to ensure adequate protection from radiation for both personnel and the public.

This paper is focused on laser research centre ELI Beamlines, which is currently under construction in Dolní Břežany, Czech Republic. The facility will be the first of the planned four that will encompass the envisioned European Extreme Light Infrastructure (ELI) Project. The ELI Beamlines facility shall develop a new generation of secondary sources for interdisciplinary applications in physics, medicine, biology and material sciences.

The facility will operate four laser systems with power ranging from 0.5 to $10 \mathrm{PW}$. The facility will offer users 13 beamlines and six large experimental halls, each having an area of between 450 and $850 \mathrm{~m}^{2}$. The laser beams will generate pulsed prompt sources of high energy electrons, photons or protons (up to several $\mathrm{GeV}$ ), producing up to $10^{12}$ particles of primary radiation per shot. Depending on the source type, the length of the pulses is expected to range from between 10 to $30 \mathrm{fs}$, with repetition rates from $0.1 \mathrm{~Hz}$ to $1 \mathrm{kHz}$. Table 1 gives an overview of the expected source terms for which the facility civil structure has been designed.

Details of the source term calculations can be found in [1] (proton and electron beams) and [2] (gamma beam). In general, electrons have been produced in the forward direction with $1^{\circ}$ divergence. Gaussian distribution of primary electron spectra with $10 \%$ energy spread has been assumed. In case of protons, rectangular energy distribution has been adopted, with $5 \%$ or $10 \%$ energy spread.

Table 1. Brief overview of the ELI Beamlines source term primary pulsed radiation produced by lasers, pulse lengths 10-30fs.

\begin{tabular}{cccc}
\hline $\begin{array}{l}\text { Energy } \\
{[\mathrm{GeV}]}\end{array}$ & $\begin{array}{l}\text { No of } \\
\text { particles/shot }\end{array}$ & $\begin{array}{l}\text { Repetition } \\
\text { rate }[\mathrm{Hz}]\end{array}$ \\
\hline \multicolumn{4}{c}{ Electron beams } \\
0.2 & $3.10^{9}$ & 1000 \\
0.5 & $6.10^{8}$ & 1000 \\
2 & $6.10^{9}$ & 10 & \\
5 & $6.10^{9}$ & 10 & \\
6.1 & $8.10^{9}$ & 10 & Associated \\
50 & $10^{10}$ & 0.1 & electrons \\
\hline \multicolumn{5}{c}{0.02} \\
0.01 & Proton beams & 0.1 \\
0.1 & $6.10^{11}$ & 1000 & 1.5 \\
0.2 & $10^{11}$ & 10 & 10 \\
3 & $10^{12}$ & 0.1 & \\
\hline \multicolumn{5}{c}{$6.10^{11}$} & Gamma beam \\
\hline max. 1.9 & $10^{9}$ & 0.1 \\
\hline \multicolumn{5}{c}{} \\
\hline
\end{tabular}

*Corresponding author. Email: olsovcova@ fzu.cz 
An overview of how the bulk shielding design has been developed for the facility, including the analysis of the consequences of introducing various penetrations, is presented. The aim of the design process was to provide efficient shielding in compliance with the ALARA principle, i.e. ensuring the safety of people by lowering their exposure, whilst optimizing the shielding in terms of cost and dimensions.

\section{Methods}

\subsection{Targeted exposure limits}

The targeted limits of effective dose obtained as a direct result of the operation of the lasers were not to exceed (per annum) $1 \mathrm{mSv}$ for personnel and $0.1 \mathrm{mSv}$ for the public. These limits are in compliance with international guidelines, e.g. [3-4], embodied into Czech national legislation through relevant Acts and Decrees, particularly [5-6].

Table 2. Radiological classification of areas, based on anticipated dose accrual rate

\begin{tabular}{lccc}
\hline & $\begin{array}{c}\text { Design target } \\
{[\mu \mathrm{S} v / \text { day }]}\end{array}$ & $\begin{array}{c}\text { Upper Limit } \\
{[\mu \mathrm{Sv} / \text { day }]}\end{array}$ & Occupancy \\
\hline R0 & $<1$ & 2.5 & high \\
R1 & $<4$ & 25 & high \\
R2 & $<25$ & 200 & low \\
R3 & no limit & no limit & no entry
\end{tabular}

R1/R3 Higher classification during the beamline operation R2/R3 Higher classification during the beamline operation

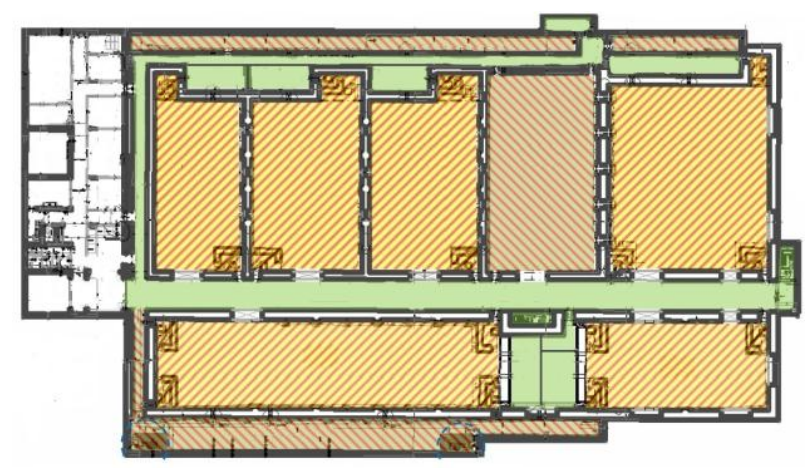

Figure 1. Layout of experimental halls with radiation zones. Legend: R0: white, R1: green, R2: yellow, R3: red, i.e. R1/R3: green with red lines, R2/R3: yellow with red lines.

\subsection{Radiation zones}

The facility is divided into four 'classifications' according to the anticipated level of hazard, taking into account expected occupancy, prompt radiation levels during beamline operation, and residual radiation levels following beam shutdown. A maximum 250 days of laser operation per year was anticipated.

Radiological classifications R0 to R3 relate to different levels of access restriction. Considering the operational regime of the facility, target and upper limit dose rates were defined for each classification, see Table 2. Shielding provisions are designed to achieve the target dose rate; however, dose rates up to the upper limit may be permitted, provided that the area where the target dose rate is exceeded is localized or inaccessible (or a combination of both) and will therefore not result in a significant increase in whole body dose.

The R0 classification applies to freely accessible areas with a maximal annual dose accrual of $0.25 \mathrm{mSv}$. However, as public members are unlikely to spend more than 100 days a year in $\mathrm{R} 0$ areas, the realistic annual dose will remain below the targeted $0.1 \mathrm{mSv} / \mathrm{y}$.

The R1 classification is assigned to high occupancy areas, accessible only to facility personnel, such as access corridors and control rooms.

The R2 classification applies to areas with moderate or low occupancy requirements, e.g. experimental halls during beam shutdown. Should there be a need for prolonged periods of occupation; implementation of local shielding will be considered to ensure that dose uptake remains acceptable and ALARA.

The R3 classification is assigned to areas where personnel access is temporarily restricted due to potentially high dose rates during the beam operation. Access will only be permitted once radiation levels are low enough to revert the area to its general $\mathrm{R} 1$ or $\mathrm{R} 2$ classification.

Figure 1 depicts the zoning for the experimental halls. Beamlines can be operated independent of the operation status in adjacent experimental halls.

\subsection{Shielding calculations}

Calculations were undertaken using Monte Carlo transport codes FLUKA 2008 [7] and the FLAIR interface [8], together with discrete ordinates code ATTILA [9]. MCNPX [10] was used to cross check FLUKA results. For Monte Carlo, computational time increases with i. a. number of particles and interactions. Although the statistics can be improved by biasing, it is inefficient for a large complicated system with many regions of interest. On contrary, for a discrete ordinates code, computational time depends on number of energy groups and defined scattering order. Further, the code calculates response everywhere in the model, not just at predefined locations. However, as Attila is not capable to simulate high energy particle transport, Monte Carlo calculations were used to generate a secondary neutron and photon source term [11]. Thus, a combination of the two methods allowed assessment of complex shielding geometries to proceed faster than could be achieved through the use of either biased or analogue Monte Carlo alone.

Ambient dose equivalents were calculated using conversion factors listed in [12] or [13] for protons, muons, neutrons above $200 \mathrm{MeV}$ and photons above $3 \mathrm{MeV}$. Materials compositions were taken from [14].

Each primary particle beam was modelled emerging from an interaction chamber through a thin window, into a basic beam dump; typically of a metal or graphite core material surrounded in concrete.

The shielding design was based on the worst 
reasonable location of the source relative to the shield that could be experienced during beamline operation. The various possible daily configurations of laser operation were considered and the most optimistic combination of laser system availability and performance was chosen as the upper limit for each given experimental hall. This ensured that doses are unlikely to be underestimated.

Residual radiation will be present in the experimental halls even when the beamline is not operating due to activation of beam dumps, shielding materials and experimental equipment by the primary sources. However, the contribution of residual radiation to doses in high occupancy areas is expected to be negligible in comparison to contributions from prompt radiation sources and therefore was not considered for both bulk shielding design and penetration analysis.

\section{Results}

\subsection{Bulk shielding}

Bulk shielding provided by the concrete structure was assessed first. Dose rates were calculated on the cold-sides of the bulk shielding. If the classification criteria for the given area were met, the bulk shielding was judged to be acceptable; otherwise, a refinement of the beam dump or the bulk shielding was performed until sufficient shielding function was demonstrated.

It was confirmed that to assure personnel dose accrual (as a consequence of laser operations) will remain lower than $1 \mathrm{mSv}$ per year, it was sufficient to build the civil structure using ordinary concrete of $2320 \mathrm{~kg} / \mathrm{m}^{3}$ density. However, in the vicinity of $3 \mathrm{GeV}$ proton source, it is necessary to use magnetite concrete (density $3450 \mathrm{~kg} / \mathrm{m}^{3}$ ) in certain locations.

Typical wall thicknesses between the experimental halls and other areas were designed as follows:

$\begin{array}{ll}\text { Control rooms } & 1.2 \mathrm{~m} \\ \text { Corridors } & 1.2-1.6 \mathrm{~m}\end{array}$

Neighbouring experimental halls $1.2 \mathrm{~m}$

Labyrinths $1 \mathrm{~m}$

Roofs (control room, labyrinth) $0.6 \mathrm{~m}$

Outside $\quad 1.6 \mathrm{~m}$

It should be noted that the wall thickness is overestimated in some areas, as thinner walls would provide sufficient protection against ionizing radiation. However, it was necessary to keep defined minimal wall thicknesses to ensure the vibration stability of the facility, necessary for a faultless laser operation.

The dose rates are driven by neutrons. Typically, the contribution of photons is less than $5 \%$. Figure 2 depicts an example of calculated dose maps for one experimental hall. Although the calculated dose rates locally exceed the criteria given in Table 2 , it is possible to relax the criteria for most of the cases, as the areas are highly localized and difficult to access. It is therefore highly unlikely that anybody would spend there their entire workdays for a whole year at such a location.

The experimental halls are located underground; therefore it is impossible for members of the public to directly access the cold side of the bulk concrete shielding. However, the space above three experimental halls is directly accessible. The concrete roof is $1.6 \mathrm{~m}$ thick. For one of the halls, this is not sufficient to reduce the dose rate above the roof to below $1 \mu \mathrm{Sv} /$ day, (calculated $4.4 \mu \mathrm{Sv} /$ day). However, the roof will be covered by $1.5 \mathrm{~m}$ of earth that would further reduce dose rates to acceptable levels. Nevertheless, further analysis of beam dumps is required to ensure dose rates remain acceptable should the earth be removed in the future.

An additional calculation was undertaken to explore the effect of removing a beam dump. Operating the $2 \mathrm{GeV}$ electron beamline under such conditions would result in a daily dose as high as $51 \mathrm{mSv}$ at the cold side, i.e. in control rooms or corridors.

Dose rates calculated by FLUKA and MCNPX were compared and found to be in satisfying agreement. Table 3 gives an example of results for several beams and cold sides of their respective control rooms.

Table 3. Example of cross-check results between FLUKA and MCNPX calculations. Presented data are dose rates at cold side of the control rooms adjoining the given experimental halls.

\begin{tabular}{rrccc}
\hline & shots/ & \multicolumn{2}{c}{ Dose rate $[\mu$ Sv/day $]$} & \\
Beam & day & FLUKA & MCNPX & Ratio \\
\hline $5 \mathrm{GeV}$ electron & 6000 & 0.3 & 0.4 & 0.75 \\
$50 \mathrm{GeV}$ electron & 100 & 0.08 & 0.07 & 1.14 \\
$500 \mathrm{MeV}$ electron & 1800 & 0.71 & 1.64 & 0.43 \\
$100 \mathrm{MeV}$ proton & 6000 & 1.32 & 1.512 & 0.87 \\
$3 \mathrm{GeV}$ proton & 100 & 4.15 & 2.63 & 1.58 \\
\hline
\end{tabular}

\subsection{Penetrations}

Once the required bulk shielding function was demonstrated, the effect of access doors, labyrinths, various technology services and ventilation penetrations upon the bulk shielding were explored. Notional detectors were placed at multiple locations on the cold-side of penetrations in order to determine doses in accessible areas.

Worst case penetrations were identified and assessed first and provided a bounding case for other penetrations. If the shielding provisions were shown to be adequate for the bounding case penetration, then other penetrations could also be judged to be acceptable.

Initially, penetrations were assessed as proposed by engineering drawings. If the calculated dose exceeded the cold-side dose criterion, different design measures (e.g. relocation or joggling) were taken, depending on their suitability and effectiveness for the given penetration function and position within the facility.

Neutron and photon fluxes (intensity, direction and spectra) generated by interactions in the beam dump were calculated by FLUKA and used to generate an anisotropic source for Attila. Contributions from primary photons (from the beam dump) to cold side dose rates were confirmed to be negligible in comparison with contributions from neutrons and secondary photons. Therefore, primary photons were not considered in this type of calculation. 


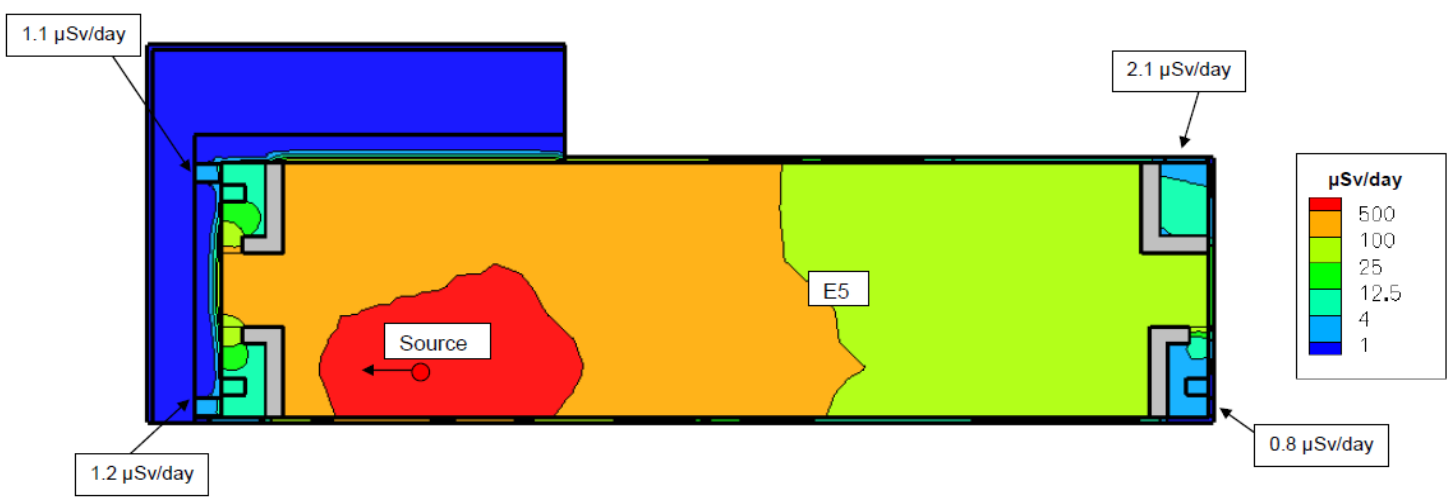

Figure 2. Calculated total dose map for an experimental hall hosting a $5 \mathrm{GeV}$ electron beam. Example of penetration analysis: in this calculation, the middle pillars in the access labyrinths are void. Dose contours provide information on the general trends of radiation transport from hot-side to cold-side areas.

A detailed analysis of the consequences of introducing various penetrations into the bulk shielding was performed. Figure 3 shows an example of penetration analysis for one of the experimental halls. The figure illustrates the complexity that multiple penetrations introduce when considering contributions from scattered radiation to cold side dose rates.

Most of the minor penetrations do not present any issues. However, cold-side dose criteria, see Table 2, can be achieved for large openings too, providing that certain measures are implemented. Generally, relocation or altering the concept of the penetration was preferred to adding extra local shielding.

In some locations, where low occupancy is expected and the dose rates are high but localized, it is possible to install a physical barrier (e.g. steel wire cage) that can be removed for maintenance when required. Also, at some locations, e.g. above control rooms, additional local shielding was recommended.

For personnel access penetrations, concrete labyrinth structures with lightly shielded doors (suitable for escape) are used. On a service gantry, running through several experimental halls, it is necessary to employ both heavy shielded doors and local access restrictions.

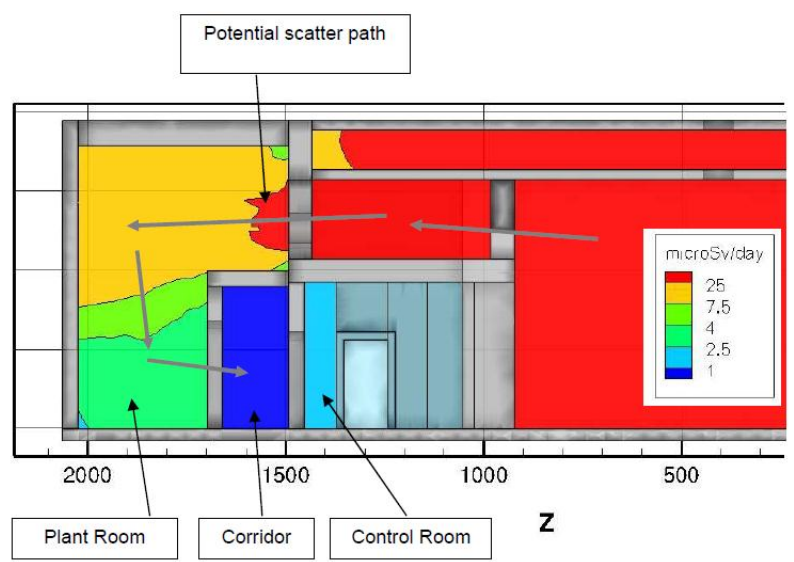

Figure 3. Example of an analysis of the radiation transport through penetrations. Radiation is leaking through the large ventilation ducts above the control room, through to the plant room and the corridor then back to the control room. Contours indicate trends only, values are approximate.
Last, but not least, all construction openings will be backfilled with polyethylene blocks corresponding to the wall thickness once the services have been routed.

\section{Conclusions}

It was possible to design bulk shielding for the ELI Beamlines laser research facility so that it will fulfil the expected shielding function, which will ensure that maximal dose accrual (as a direct result of laser operation) to individual personnel will be lower than $1 \mathrm{mSv} /$ year. Operations not requiring manual intervention will be performed remotely from the control rooms so far as reasonably practicable. Interlock systems will be installed to restrict access to the specified areas.

The effect of various penetrations on cold side doses were analysed case by case and appropriate measures taken. As the dose rates are driven by neutrons; all the remaining construction openings will be backfilled with polyethylene blocks after installation of services.

\section{Acknowledgements}

This work benefitted from support of the Czech Republic's Ministry of Education, Youth and Sports and of the Academy of Sciences of the Czech Republic to the ELI-Beamlines project, registered under No. CZ.1.05/1.1.00/02.0061.

\section{References}

[1] A. Ferrari, E. Amato, D. Margarone, T. Cowan and G. Korn, Radiation field characterization and shielding studies for the ELI Beamlines Facility, Applied Surface Science, (2012), DOI: 10.1016/j.apsusc.2012.09.105.

[2] Y. Hayashi, A. Fukumi, K. Matsukado, M. Mori, H. Kotaki, M. Kando, L. M. Chen, I. Daito, S. Kondo, S. Kanazawa, A. Yamazaki, K. Ogura, M. Nishiuchi, M. Kado, A. Sagisaka, S. Nakamura, Z. Li, S. Orimo, T. Homma and H. Daido, Estimation of photon dose generated by a short pulse high power laser, Radiation Protection Dosimetry 121 
(2), (2006), pp. 99-107.

[3] Council Directive 96/29/EURATOM, Basic safety standards for the protection of the health of workers and the general public against the dangers arising from ionizing radiation, 13 May 1996.

[4] IAEA, Radiation protection and safety of radiation sources: International Basic Safety Standards Interim Edition, General Safety Requirements Part 3, No. GSR Part 3, (2011).

[5] Act No. 18/1997 Coll., on Peaceful utilization of nuclear energy and ionizing radiation.

[6] Decree No. 307/2002 Coll., on Radiation Protection as amended in 499/2005 Coll.

[7] A. Fasso, A. Ferrari, J. Ranft and P. R. Sala, FLUKA-2008: A Multi-particle Transport Code, CERN-2005-10 (2005), INFN/TC_05/11, SLAC-R-773.

[8] V. Vlachoudis, FLAIR: A powerful but user friendly graphical user interface for FLUKA, Proc. Int. Conf. on Mathematics, Computational Methods \& Reactor Physics (M\&C 2009), Saratoga Springs, New York, (2009).
[9] ATTILA 7.0.1, Transpire Inc. Attila Radiation Transport Software Support Tips: "Anisotropic Point Source File”, (2007), www.transpireinc.com.

[10]X-5 Monte Carlo Team, MCNP - A General Monte-Carlo N-Particle Transport Code, Version 5, LA-UR-03-1987, Los Alamos National Laboratory, April $24^{\text {th }},(2003)$.

[11]L. MacFarlane, Monte Carlo flux profile to Attila Anisotropic point source method, Nuclear Technologies Technical Guide NT/R964, Issue 1, (2011).

[12]ICRU 57, Conversion Coefficients for Use in Radiological Protection against External Radiation, (1998)

[13]M. Pellicioni, Overview of fluence-to-effective dose and fluence-to-ambient dose equivalent conversion coefficients for high energy radiation calculated using the FLUKA code, Radiation Protection Dosimetry 88 (4), (2000), pp. 279-297.

[14]CRC Handbook of Chemistry and Physics, 82nd Edition, (2001-2002). 